\title{
Design and development of Ionization Profile Monitor for the Cryogenic sections of the ESS Linac
}

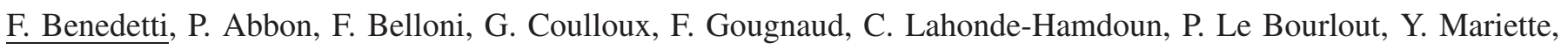 \\ J. Marroncle, J.P. Mols, V. Nadot, L. Scola and G. Tauzin \\ CEA Saclay, Institut de Recherche sur les lois Fondamentales de l'Univers \\ Gif-sur-Yvette, France \\ R. Tarkeshian and C. Thomas \\ European Spallation Source ERIC \\ Lund, Sweden
}

\begin{abstract}
Saclay CEA/IRFU is working for the delivery of five Non-Invasive Profile Monitors in the frame of the in-kind contribution agreement signed with the European Spallation Source. Neutrons will be produced by spallation reactions of $2 \mathrm{GeV}$ proton beam impinging on a Tungsten target. To accelerate protons a powerful linear accelerator of $5 \mathrm{MW}$ is under construction. Diagnostic devices are mandatory tools for the tuning and protection of the machine. The non-invasive profile monitors provide a measurement of the beam profile in transverse directions to the beam propagation. This project raises several physical and technical challenges including low signal detection of ions or electrons, profile distortions induced by the beam Space Charge effect and non-uniformities of electric field. Simulation and model of the critical aspects of the detector have been performed in order to prove the performance and the feasibility of the detector. A series of prototypes has been built with different readout types, and tested in real conditions at the $3 \mathrm{MeV}$ proton accelerator IPHI. All of them show some advantages and drawbacks revealed by the tests in real beam conditions. In this paper we present the results of the tests for the various configuration readout systems to agree with the model and simulation of the detector. In concluding remarks, we will discuss the performance of the prototypes and point out the camerabased one to be the more suitable for the final design.
\end{abstract}

Index Terms-Beam diagnostic, Linear proton accelerators, MCP, Strip detector, Particle beam measurements

\section{INTRODUCTION}

$\mathbf{T}$ HE European Spallation Source (ESS) will be a European research infrastructure dedicated to neutronic science. The source is currently under commissioning at the ESS site in Lund, Sweden, and will be the brightest pulsed neutron source. The production of neutrons is ensured by the spallation process: high energy protons will impinge on a tungsten target. To accelerate the protons a powerful $2 \mathrm{GeV}$ linear accelerator is being built. The accelerator can be schematized in two parts. The first part accelerates the beam up to $90 \mathrm{MeV}$ by mean of conventional room temperature RF cavities. Then a

The project was supported by the French in-kind contribution to ESS (AIK 7.3).

F. Benedetti is with the Département d'Électronique des Détecteurs et d'Informatique pour la Physique, Commissariat à l'Énergie Atomique et aux énergies alternatives, Saclay, 91400 France, (email: florian.benedetti@cea.fr). cold part using superconducting cavities cooled with liquid helium is used to reach the highest energies. An overview of the ESS linac is shown in Fig. 1, and Table I gives its main characteristics.

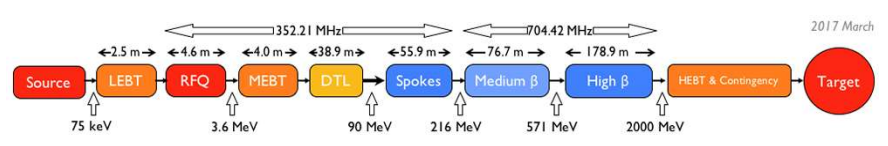

Fig. 1. A simplified representation of the ESS linac. The IPMs will be installed in the cryogenic part (blue).

At ESS, both invasive and non-invasive profilers will be installed along the accelerator. The beam profile will be also recorded at the target location and upstream of the beam dump [1]. The invasive measurements are mainly done with wire scanners. These devices cannot handle the huge beam peak power of ESS at nominal conditions (125 MW), and will be only used at low beam duty cycle [2]. Therefore, Non invasive Profile Monitors (NPM) will take over for higher beam power. In fact, the NPM refers to two types of devices depending on the detection principle. Fluorescence Profile Monitors (FPM) [3] are foreseen in the "warm" parts of the accelerator whereas Ionization Profile Monitors (IPM) will be implemented exclusively in the cryogenic part of the accelerator. Five pairs of IPMs are foreseen at several locations.

TABLE I

ESS LINAC CHARACTERISTICS

\begin{tabular}{ll}
\hline Characteristic & Value \\
\hline Energy & $2 \mathrm{GeV}$ \\
Pulse current & $62.5 \mathrm{~mA}$ \\
Pulse duration & $2.86 \mathrm{~ms}$ \\
Repetition rate & $14 \mathrm{~Hz}$ \\
Duty cycle & $4 \%$ \\
Power (peak) & $5 \mathrm{MW}(125 \mathrm{MW})$ \\
Radio Frequencies & $352.21 \mathrm{MHz}$ \\
& $704.42 \mathrm{MHz}$ \\
\hline
\end{tabular}




\section{IONIZATION PROFILE MONITORS}

\section{A. State of art}

The first use of IPMs dates back to the 1960s [4]. In the 90s, the raise of the MicroChannel Plates have permitted to measure profiles [5], [6] in more critical working conditions. The IPM method is now mature and used in several installations [7][10]. Recently, the interest in semiconductor detectors has grown [11] and first results looks promising [12].

The different technologies of detection, presented just before, have been reviewed in order to select the most efficient one with respect to the ESS requirements.

\section{B. Principle of operation}

An Ionization Profile Monitor (IPM) is a non-invasive detector that measures the transverse profile of a beam. Its principle of operation is shown in Fig. 2 and can be summarized in 3 main steps:

1) Beam protons pass through the vacuum, inducing ionizations of the residual gas molecules: electron/ion pairs are created.

2) Inside the IPM, a strong electric field drives electrons or ions towards a segmented readout system.

3) The profile is reconstructed in one transverse direction. For a complete profile, a pair of IPMs, rotated by $90^{\circ}$ with respect to each other, is mandatory.

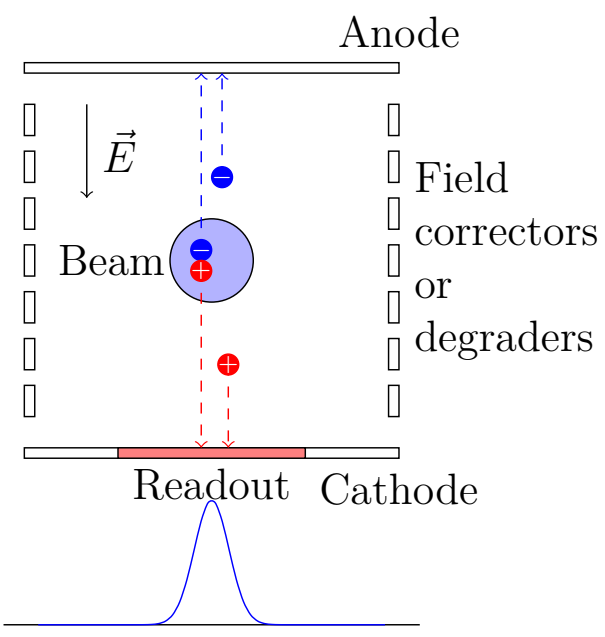

Fig. 2. Visual explanation of how an IPM works. The electric field between the electrodes can be reverted by inverting the polarity, giving the possibility to detect ions or electrons. Field correctors or degraders, left and right, improve the field uniformity.

One can note that an IPM forces the charge collection with an electrical field, whereas a FPM is passive and detects within a certain solid angle. On the other hand, the implantation of IPMs is more complex since it operates in vacuum.

Simulations were done in order to check the feasibility of the IPM design for the given ESS conditions. Each simulation focused on the following hot topics:

- Quantification of the ionization signal in terms of number of produced electron/ion pairs for ensuring that the signal is sufficiently high for reconstructing a profile per pulse beam, despite of the low gas pressure and relatively small ionization cross section at high proton energy.

- The extraction field must be as uniform as possible in order to lead efficiently and correctly the ionization byproducts toward the readout.

- The space charge effect induced by the beam and the initial momentum of ionization electrons/ions, which may distort the profile, must be evaluated.

- The choice of an efficient readout technology which must match the ESS working conditions.

All these points will be presented in the next sections.

\section{ELECTRON/ION RATES}

The IPMs rely on the by-product collection of the ionized residuals gas. The number of ionized particles gives the signal strength which has to be compliant to the readout sensitivity. Therefore, the number of ionization particles, that are created by the beam itself along the residual gas, must be evaluated.

\section{A. Calculation using Bethe model}

For heavy charged particles like protons, the main interaction is due to electromagnetic interactions of the incident particle with the orbiting electrons of the medium. The Bethe equation describes the mean rate of energy losses per distance unit by a heavy charged particle as follows [13, p. 446]:

$$
-\left\langle\frac{d E}{d x}\right\rangle=K \rho \frac{Z}{A} \frac{z^{2}}{\beta^{2}}\left[\frac{1}{2} \ln \left(\frac{2 m_{e} \beta^{2} \gamma^{2} T_{\max } c^{2}}{I^{2}}\right)-\beta^{2}\right]
$$

where $K$ is a constant factor defined by $K=4 \pi N_{a} r_{e}^{2} m_{e} c^{2}$, $r_{e}$ is the classical electron radius, $m_{e}$ is the electron mass, $N_{a}$ the Avogadro constant and $c$ the speed of light in vacuum. $Z$, $A$ and $\rho$ are respectively the atomic number, the mass number and the density of the given medium. The mean excitation energy $I$ is the only non-trivial variable in the Bethe equation [14]. The maximum transfer energy for one collision $T_{\max }$ is given by the following equation:

$$
T_{\max }=\frac{2 m_{e} \beta^{2} \gamma^{2} c^{2}}{1+\frac{2 \gamma m_{e}}{M}+\left(\frac{m_{e}}{M}\right)^{2}}
$$

where, $M$ and $m_{e}$ are respectively the incident particle and electron masses. The $\beta$ and $\gamma$ variables have their normal significance as Lorentz factors.

When the medium is a mixture of several compounds, its mean stopping power needs to be calculated as the sum of the mean stopping power of its components weighted by their mass proportion. By introducing $W$, the average energy for producing an ion/electron pair in a medium [15], [16], one can estimate the number of ion/electron pairs created in a given readout length $\Delta x$ of materials as:

$$
N_{\text {total }}=\sum_{n=\text { First }}^{\text {Last }} \frac{\left\langle\frac{d E}{d x}\left(\rho_{n}, I_{n}, A_{n}, Z_{n}\right)\right\rangle}{W_{n}} \Delta x
$$

The Bethe formula can be implemented in a $\mathrm{C}++$ code once the composition of the medium and the $I$ value of each compound is known. The expected pressure in the cryogenic part at ESS is around $10^{-9} \mathrm{mbar}$, and the gas composition is given by the following: $79 \% \mathrm{H}_{2}, 10 \% \mathrm{CO}, 10 \% \mathrm{CO}_{2}$ and $1 \% N_{2}$ 


\section{B. Calculation using PAI model}

The Garfield++ software [17] also has been used to compute the number of primary ionizations. This software is normally intended for the modelization of gaseous detectors. It allows to simulate the creation of electron/ion pairs due to the ionization of gas by an incident particle, the transport and amplification of these electrons in the gas and the signal induced on a readout plane. In our case, we simulated only the pair creation in the residual gas. For this step Garfield++ uses Heed ++ [18], a C ++ code that implements the photoabsorption and ionization (PAI) model [19].

Table II summarizes the results of the two previous calculations done for different IPM positions along the ESS linac, and the factor ratio between the methods.

TABLE II

COMPARISON OF EXPECTED NUMBER OF ELECTRON/ION PAIRS PER $\mathrm{cm}$ BETWEEN CALCULATION USING BETHE EQUATION AND RESULTS FROM GARFIELD++.

\begin{tabular}{llll}
\hline Energy & $N_{\text {Bethe }}$ & $N_{\text {garfield }}$ & Factor \\
\hline 97.2 & 100210 & 52537 & 0.52 \\
231.4 & 54970 & 27463 & 0.50 \\
278.9 & 49160 & 26124 & 0.53 \\
315.8 & 45850 & 23769 & 0.52 \\
628.3 & 33600 & 17522 & 0.52 \\
\hline
\end{tabular}

\section{Limitations}

The estimation strongly depends on various vacuum parameters that have not been measured yet. In consequence, the calculated values give only a coarse approximation. Also, the measurement of the $W$ parameter includes the delta ray (or secondary ionization) electrons, hence the $W$ value is biased [4], [13] for the case at hand, since the IPMs work at very low pressure. The real $W$ values may be higher than the ones given in references, therefore the real number of ionization pairs may be 2 to 4 times lower than the expected number.

\section{PROFILE DISTORTIONS}

\section{A. Extraction field}

The IPMs can be seen as parallel plate detectors. In an ideal case, these plates are infinite sized. The extraction field is then completely oriented in a single direction, normal to the detection plane and the projection of the profile on this plane is perfect. In reality, the plates have finite dimensions, comparable to the gap between the two electrodes. In these conditions the field is no more uniform. In addition, the crossinteraction between the electric fields of two close by IPMs is very strong. The non-uniformity of the electric field is very problematic because it creates mirage effects and prevents the correct measurement of the beam profile. To overcome this effect, two solutions have been considered:

- Using field correctors or field degraders [10]. This is done by placing conductors on each side. Each corrector is set to a certain potential in order to constrain the field.

- Putting grounded conductors between the two IPMs [10] to protect against the IPM cross-interaction.
COMSOL has been mainly used for the simulations of extraction fields, and a particle tracking algorithm has been implemented in $\mathrm{C}++$. The code integrates the motion of particle due to the Lorentz force:

$$
\vec{F}=m \cdot \vec{a}=q \cdot(\vec{E}(\vec{r}, t)+\vec{v} \times \vec{B}(\vec{r}, t))
$$

Fig. 3 shows an example of results given by the particle tracking. Without the corrections, the reconstructed profile is $35 \%$ wider than reality. The shift in position is due to the cross interaction between the two IPMs. When corrections are enabled, the obtained transverse profile is much better: the error on the profile width is only $0.4 \%$ and there is no more shift in position.

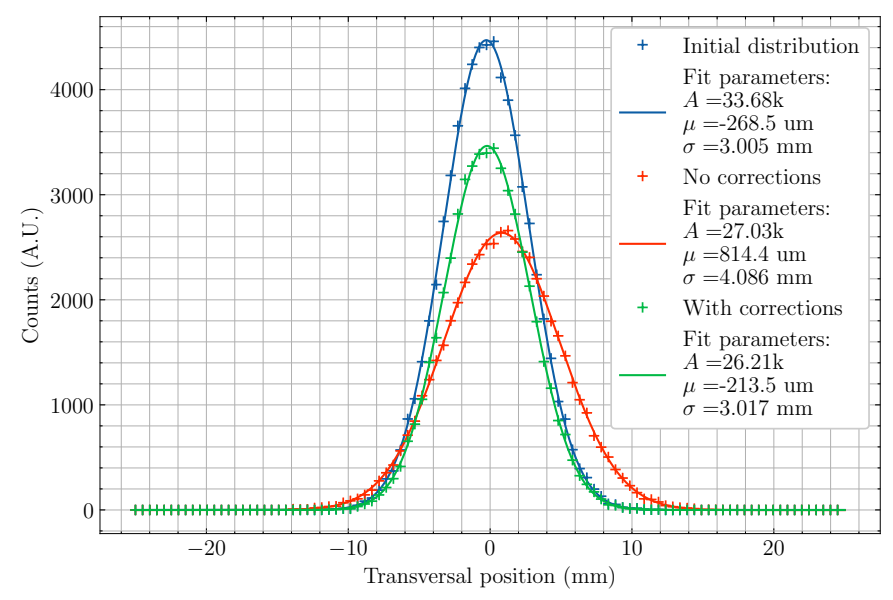

Fig. 3. A simulation of profile measurement with particle tracking for two real field configurations: without correction and with corrections. The initial proton (blue) beam is assumed to be Gaussian with $\sigma_{\text {beam }}=3 \mathrm{~mm}$ (blue). Without correction, a shift of $1 \mathrm{~mm}$ and a broadening of $35 \%$ are observed (red). The corrections allows to negate the two previous distortions (green).

\section{B. Space Charge effect}

In the context of IPMs, the Space Charge effect refers to the influence of the beam itself on the profile measurement. An ESS bunch is about $10^{9}$ protons, therefore bunches generate a strong electromagnetic field while moving along the accelerator, and the electrons or ions are affected by this electromagnetic field while drifting to the read-outs in a IPM.

The estimation of this electromagnetic field is necessary to ensure that the profile measurement is not too strongly affected by the space charge effects. For this reason, a simulation code has been developed conjointly by ESS and CEA/Saclay. The code computes the electric field of a 3D Gaussian distributed bunch in the moving frame $\bar{K}$ (rest frame for bunches) [20]. The electric field is transformed into an electromagnetic field in the laboratory frame $K$ (rest frame for IPMs) by the Lorentz transformations of electromagnetic fields:

$$
\boldsymbol{E}=\left(\begin{array}{c}
\gamma \bar{E}_{x} \\
\gamma \bar{E}_{y} \\
\bar{E}_{z}
\end{array}\right), \boldsymbol{B}=\left(\begin{array}{c}
-\gamma v_{b} \frac{\bar{E}_{y}}{c^{2}} \\
\gamma v_{b} \frac{\bar{E}_{x}}{c^{2}} \\
0
\end{array}\right)
$$

Then, the motion of the electrons or ions is integrated thanks to the equation (4). 


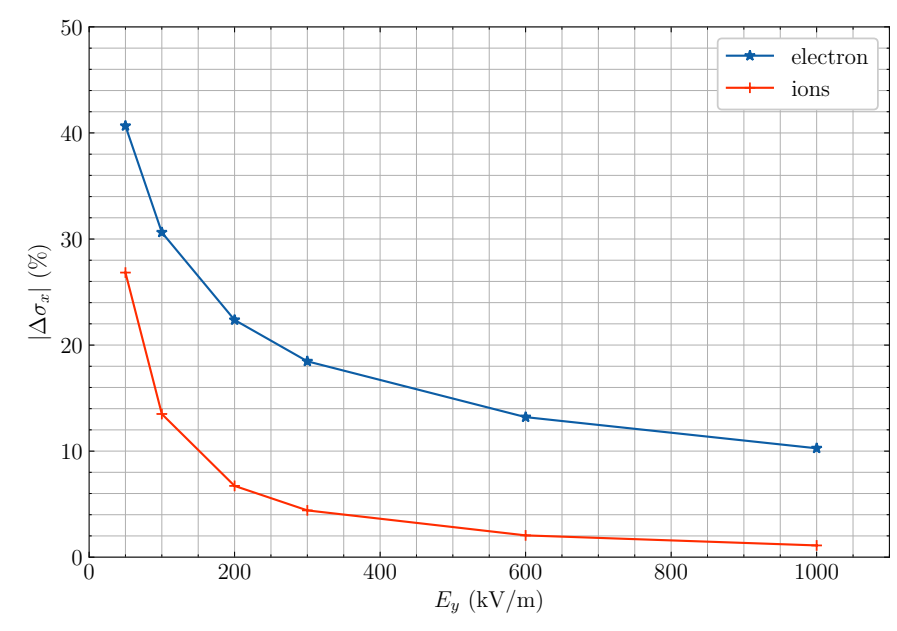

Fig. 4. Error on the profile measurement, for electrons and $\mathrm{H}_{2}^{+}$ions, due to space charge effects versus the extraction field. The beam is assumed to be a Gaussian with $\sigma_{x}=\sigma_{x}=\sigma_{x}=2 \mathrm{~mm}$

Using the code, it has been shown that space charge effect can be partially compensated by a high extraction field value. It has been seen that profiles from electrons seem to be more affected by space charge effect than from ions. In the Fig. 4, a general example of the ESS beam size measured from profiles obtained with ions and electron is presented, and for various values of the electric field strength. The beam size has been chosen to be $3 \%$ smaller than the nominal value. The values of the field strength used in the simulation are chosen in a range that is technically achievable. Within that range it can be seen that the minimization of the space charge effect is limited. It is also clear that the effect is stronger for electrons than for ions.

In the case of ESS beam, the ions and electrons feel a permanent electric field from the HV cage of the IPM, and an variable field due to the bunched structure of the beam. The duty cycle of the variable beam plays a role in the distortion of the profiles. In the case of electrons, due to their small mass, they will reach the detector within the passage of one bunch. Therefore, they feel the combined forces most of the time along their path. In the case of the ions, it takes the time of many bunches for them to reach the detector. Hence, the forces from the bunch are weighted by the duty cycle of the varying electric field. For the case of ESS, the bunches are $3 \mathrm{ps}$, and the period between them is $2.84 \mathrm{~ns}$. So the integrated force seen by the ions has less strength than in the case with electrons.

The expected uncertainty for the beam size measurement is $10 \%$. The presented case shows the impact of the space charge can be strong, but yet the retrieved values are still within the requirements for the measurement uncertainty. Larger beam sizes are associated with weaker space charge effect, hence, the designed instrument is expected to deliver reliable information on the ESS beam profile and size.

A workaround to totally counterbalance even more the space charge effects consists in adding a magnetic field parallel to the extraction field with a magnet [8]. However, this solution is not possible at ESS since the available space is too limited.
Consequently, the profile measurements will be performed with ions.

More detailed reviews of the simulations of space charge effects for the ESS IPM project are available here [21], [22].

\section{Initial momentum}

A first approximation of the initial speed of ions can be done thanks to the distribution of Maxwell-Boltzmann.The speed of the fastest ion $H_{2}^{+}$is below $5000 \mathrm{~m} / \mathrm{s}$. A field of few hundred volts per centimeter is more than enough to compensate this effect, therefore the thermal motion for ions can be completely neglected.

Garfield++ can be used again since it also gives the energy distribution of the ionization electrons as well as the angular distribution. The energy distribution spread up to $\mathrm{keV}$ range but most probable energies are below $50 \mathrm{eV}$. The ions has supposed to be emitted with the opposite momentum. In consequence, the beam measurement with electrons is also more affected by the initial momentum than the measurement with ions. This enforces even more the necessity to measure the profile with ions at ESS.

\section{REAdout TECHNOLOGIES}

\section{A. Strips}

Conductive strips is the simplest method to implement. Electrodes are etched on a PCB with a thin layer of copper. When electrons or ions move towards the electrodes, they induce a current that can be calculated by mean of RamoSchockley theorem [23], [24]. The induced current is then integrated by a front end electronic.

A COTS (Component Of The Shelf) front end electronics has been selected for this purpose: the CARAMEL card from the FASTER platform. This solution is developed by the LPC laboratory at Caen in France. CARAMEL is a charge digitizer card based on the DDC316 chip from Texas Instruments. Each card provides 32 input channels and two cards can be plugged in an AMC mother board. The mother board is compatible with $\mu$ TCA crates.

This method is very robust and radiation hard since strips are passive components. However, strips alone can not be used when the signal is too low, and it is necessary to find a way to amplify the signal.

\section{B. $M C P s$}

A MicroChannel Plate (MCP) generates electrons from incident particles [25]. When a particle hits the MCP hole entrance, secondary electrons are emitted. Due to difference of potential, secondaries are drawn towards the channel output and strike hole walls again, creating more and more electrons. Then, electrons are collected on a detection plane. The detection plane can be a phosphorus screen which converts electrons to visible photons or conductive strips. Both solutions have been implemented.

A vision system is necessary to record light from the phosphorus screen. A camera with a lens should be sufficient in the case at hand. High resolution is not mandatory, so pixels 
could be relatively big in order to increase light collection and dynamic range. Sony IMX249 fits well with these prerequisites, so the FLIR Blackfly-PGE-23S6M-C camera has been selected.

Unfortunately MCPs suffer of aging effect, the gain is not stable and decreases over the time. Therefore, a calibration must be performed to correct the gain from time to time.

\section{Silicon detectors}

The last solution consists of a pixelated silicon detector. When a charged particle passes through the silicon it deposits its energy and electron/hole pairs are created. Then, the charge carriers drift in the semiconductor due to the bias voltage, and the induced signal is read by an embedded electronic. A single particle may create thousands of secondaries, therefore silicon detectors are very sensitive and interesting for IPMs.

The use of silicon detectors seems very promising but detection is not assured for ions at low energies since the stopping range is comparable to the deadlayer of silicon detectors. The feasibility of silicon detection for IPM with ions had to be checked. To this end, a small test bench has been developed and installed in a ion-implant facility: IRMA [26]. The test has been done with a tailored TimePix [27], [28] detector, kindly provided by the CERN-BI team.

The most crucial result of the test at IRMA is shown in Fig. 5. The detection seems possible at $15 \mathrm{keV}$, however at slightly lower energy $12 \mathrm{keV}$, the signal completely vanishes. Therefore, this solution has been discarded since it give almost no margin for error.
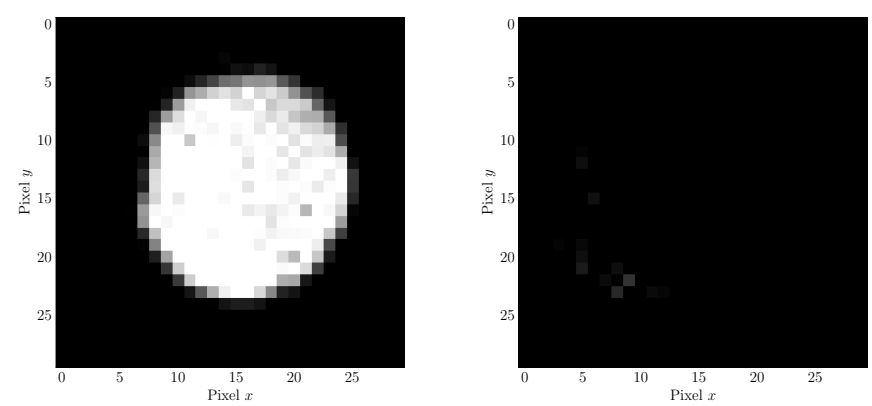

Fig. 5. Images of Time over Threshold signal from a TimePix chip for a incident $\mathrm{H}_{2}^{+}$beam at $15 \mathrm{keV}$ (left) and $12 \mathrm{keV}$ (right).

\section{PROTOTYPES DESIGN AND TEST SETUP}

\section{A. IPM and test bench design}

The simulations presented in the previous sections show that the profile measurements with IPMs may match the ESS requirements. However, some critical points, particularly the choice of the readout, were not fully clear, so the feasibility had to be proven experimentally. For this purpose three prototypes have been manufactured. The design of the prototypes is independent of the readout technology, so the readout can be changed with only few operations. A prototype using a MCP is shown in Fig. 6

A test bench has been developed in order to validate the prototypes. The bench can be split into two different independent

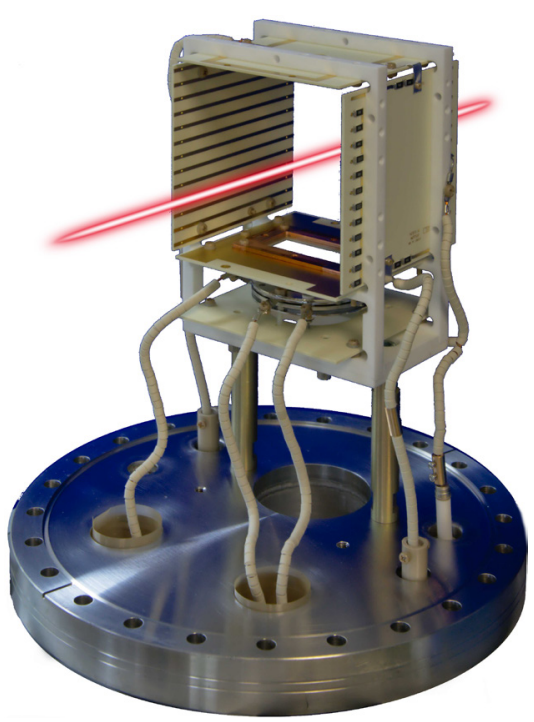

Fig. 6. One of the IPM prototypes. Here the readout is a MCP, visible through the rectangular slit. The copper lines on each side are the field correctors.

parts. The first part (upstream) tries to mimic the ESS LWU chamber inside which two IPMs can be inserted. The idea is to be close to the ESS conditions in term of high voltages and electrical fields. The second part (downstream) offers one more IPM slot and two viewports for reference measurements in order to compare with the IPMs ones. Two solutions have been considered for the reference measurements: fluorescence profile monitors (FPMs) and scintillating screens. The IPMs can be mounted independently in $\mathrm{Y}$ or $\mathrm{X}$ direction thanks to their design, thus it is even possible to measure the same profile direction with all three IPMs.

\section{B. Control System}

The whole system is almost fully compatible with the version 3.16 of EPICS base. The PointGrey GigE cameras are well supported by the AreaDetector module. A custom plugin, developed by ESS, performs a gaussian fit on every image of the profile. Raw images are saved into HDF5 files. This format allows to combine various datasets together, for instance the raw IPM images with some beam information. Since all high voltage power supplies have their own SPCI Ethernet interface, a simple softIOC with StreamDevice is enough to control and monitor them. Three OPIs have been developed in order to control cameras, power supplies and a motor (geobrick) to move scintillating screens vertically to intercept the beam or to be safely moved far from it. These OPIs run under the BOY module of the ESS Control System Studio version 4.5. An Archiver Appliance records and saves slow process variables from the power supplies, the vacuum systems and the accelerator.

\section{IPHI accelerator}

IPHI is a high intensity linear proton accelerator located at CEA/Saclay. This project started in the late of 90's [29] but protons were accelerated up to $3 \mathrm{MeV}$ in April 2016 [30]. 
Proton plasma is created by an electron cyclotron resonance source, and transported toward a radio frequency quadruple (RFQ) by a low energy beam transport line. An iris ensures a fine tuning of the current, and two solenoids focus and filter the plasma before the injection in the RFQ. Then, the protons are accelerated up to $3 \mathrm{MeV}$ and bunched with a frequency of $352 \mathrm{MHz}$ by the RFQ. A medium energy beam transport line, downstream from the RFQ, contains focusing elements, steerers, dipole magnet and beam diagnostics. The dipole magnet can distribute the protons over two beam lines.

The main line has a dedicated beam stop of $300 \mathrm{~kW}$, allowing the commissioning of the accelerator at high intensity and duty cycle. The secondary line is more modular but restricted to lower intensity and duty cycle (few hundred Watt). In 2018, this line was open for external user experiments [31].

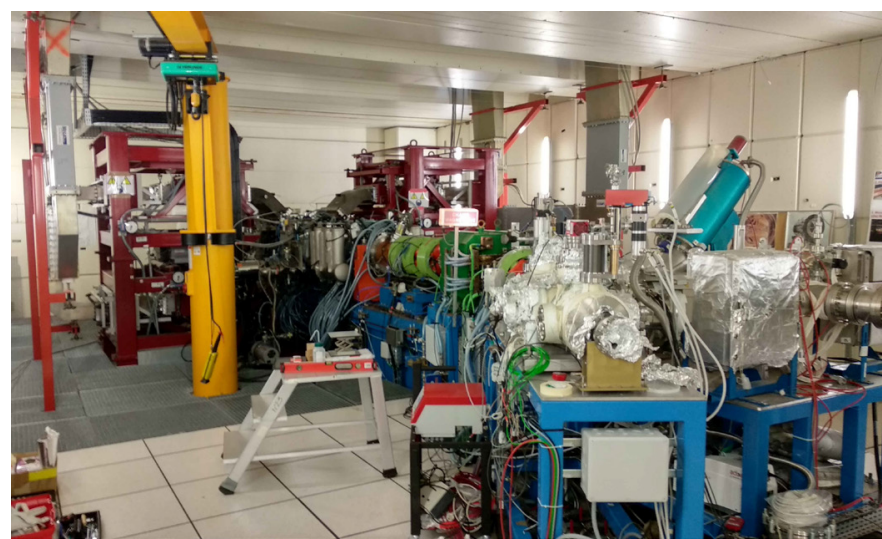

Fig. 7. Picture of the IPHI installation. The IPM test bench is also visible in the foreground.

\section{RESULTS FROM IPHI TESTS}

\section{A. First measurements}

Two test campaigns were accomplished at IPHI. In the first campaign, the correct operation of the IPMs was checked, the beam was finely tune and first measurements were performed. In the second campaign, further studies were carried out and the first results were confirmed. During both campaigns, the beam was tune to minimize its divergence in the dispersive plane. Both types of IPM have been working in both campaigns.

The optical IPM gives directly an image of the beam in longitudinal and transverse direction. The processing of data is done as follow. First, dead pixels are removed and the image is cropped to a region of interest. Then, a FFT filter is applied for reducing the noise due to sensor if necessary. The profile is reconstructed by summing all pixels in the longitudinal direction. Fig. 8 shows an image acquired from the optical IPM. One can see the shadow of a grid that is placed right in front of the MCP input. The grid has a pitch of $450 \mu \mathrm{m}$ and a wire size of $50 \mu \mathrm{m}$. This gives an idea of the resolution that can be achieved with the optical IPM.

The strips measure the profile in only one transverse direction. The data processing is different because the acquisition is done continuously. First, the pedestals are removed and

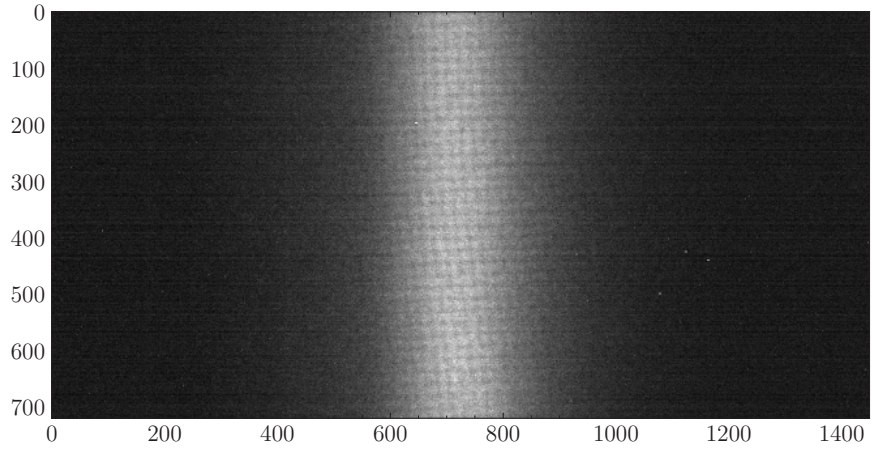

Fig. 8. An example of a beam projection image recorded by the camera. The image covers an area of $38 \mathrm{~mm}$ by $18 \mathrm{~mm}$.

a search is performed in the whole dataset to find events corresponding to a pulse. Fig. 9 shows the same profile measured by both IPMs.

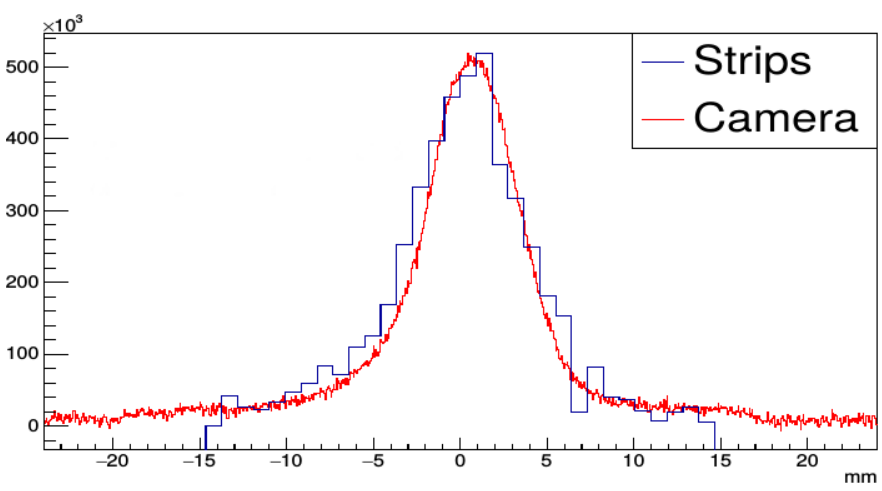

Fig. 9. Superposition of the same beam profile recorded with the strips and optical IPMs.

\section{B. Comparison with IPHI diagnostics}

Unfortunately, there is no profile measurement on the IPHI beam line, therefore the measurement of the beam profile can not be compared to an existing diagnostic. However, a complete system of Beam Profile Monitors (BPM) and AC Current Transformer (ACCT) are installed on IPHI. The beam can be also scanned with electrostatic steerers and the current is tuned by an iris aperture close to the source. An IPM is able to measure position and current of a beam, even if it is firstly intended for profile measurement.

An example of beam scanning is illustrated in Fig. 10. The sharp steps are due to steerers whereas the small transitions between two steps are the variations of beam position from pulse to pulse. This is not a desirable effect and it has limited the measurement of the position resolution. The reason of this effect is not clear and may be due to the short pulse duration time used during the tests.

The same can be done for the beam current. The signal is measured at several beam currents for a fixed IPM gain. IPMs response is very linear over an important current range. At IPHI, the number of particles collected by the MCP is an order of magnitude higher than the one expected at ESS, and no 


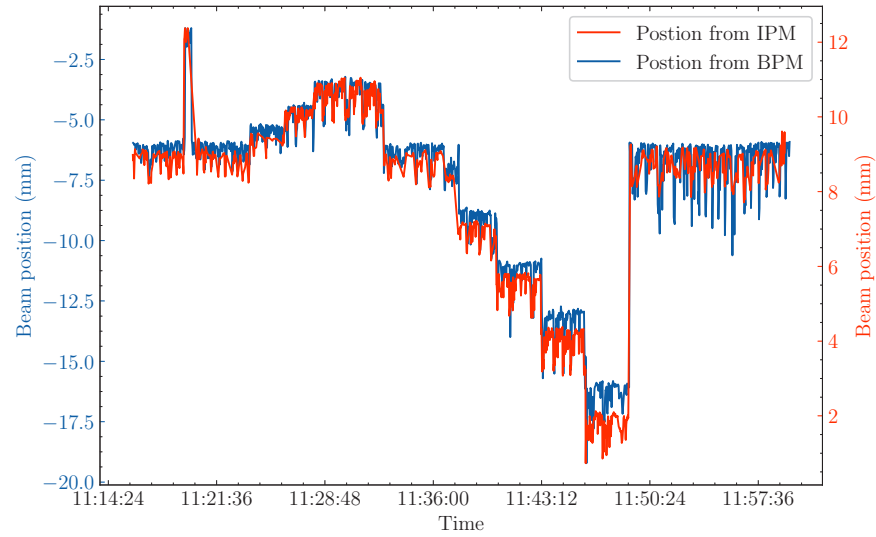

Fig. 10. Beam position over the time, measured with the BPM and the optical IPM. A steerer has been used to move the beam (step transitions). However, small variations between two steerer steps were not expected.

channel saturation has been observed on the MCP signal. This means that a single stage MCP will not suffer of saturation effect at ESS.

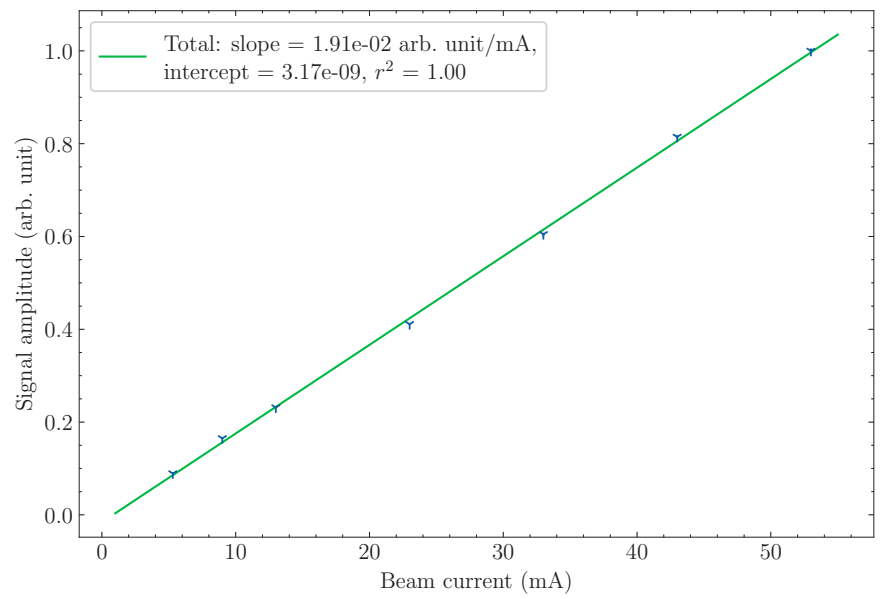

Fig. 11. Evolution of the signal with the beam current. The evolution has a good linearly over an important current range, and no saturation has been observed.

\section{Extrapolation to ESS condition}

One of the important studies conducted at IPHI is the estimation of the detection limit. The objective is to determine which readout can detect the signal in ESS conditions. However the conditions at IPHI are different from the ones of ESS. The proton energy at IPHI is $3 \mathrm{MeV}$, and according to Bethe equation, the cross section is 60 times higher compared to $2 \mathrm{GeV}$ proton. The vacuum level is also higher by one or two decades. To compensate for these two factors it is possible to reduce the beam current and pulse duration.

For bare strips readout, the number of charges collected was measured for several beam currents, and the intersection of the sensitivity curve with the noise level gives a rough idea of the limit of detection. However, this method greatly underestimates the integration noise of the electronics because the IPHI pulses are very short. According to the first results, the detection limit is just above the most favorable case of ESS. Measuring with bare strips seems difficult in ESS conditions.

Unlike the strips IPM, it is almost impossible to quantify the number of primary particles without a complete calibration of the MCP. Therefore, the extrapolation is done by calculating the Bethe formula with respect to the beam parameters and vacuum conditions measured at IPHI. The beam current and the pulse duration were set to their lowest values, respectively $0.5 \mathrm{~mA}$ and $50 \mu \mathrm{s}$. The pressure was about $4 \cdot 10^{-8} \mathrm{mbar}$ In these condition the Bethe scaling is equal to one for the worse ESS case $(628.3 \mathrm{MeV})$, and the resulting profile is shown in Fig. 12. The measurement may be possible at ESS even with a single stage MCP.
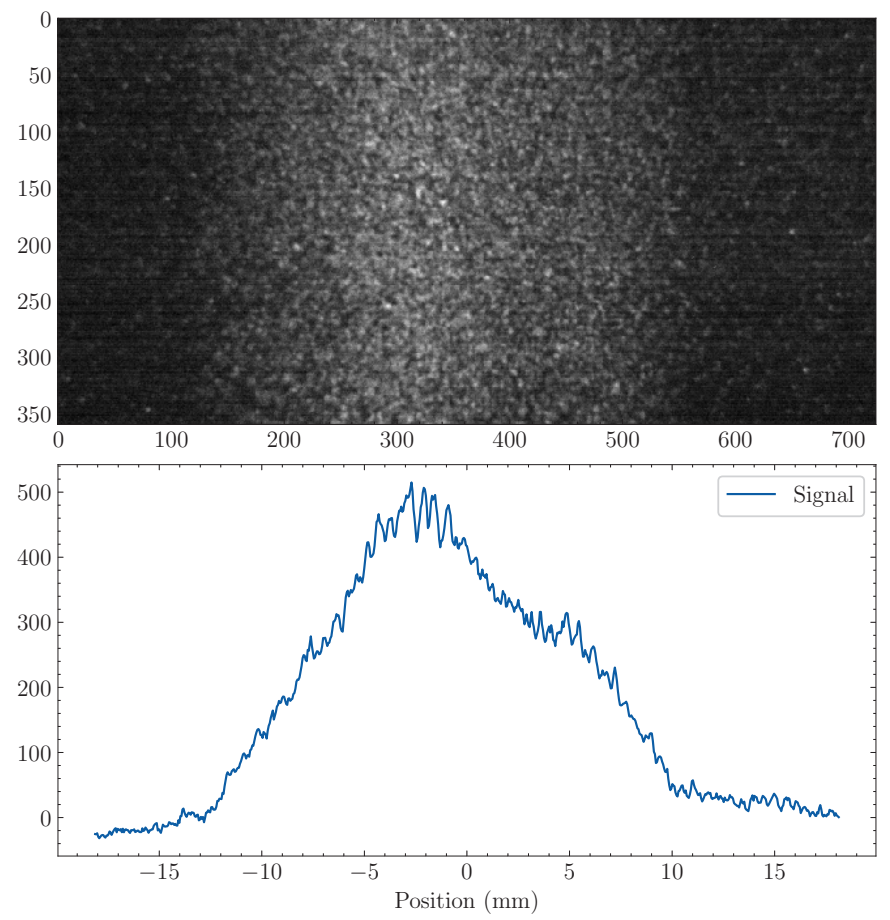

Fig. 12. Image of the beam profile at the lowest current and pulse duration possible at IPHI $(0.5 \mathrm{~mA}, 50 \mu \mathrm{s})$. In these conditions, the signal is close to the one expected at ESS for the worst case. Note that IPHI was not designed to work a such low current.

\section{CONCLUSION}

A complete study of all the critical key points concerning the feasibility of this detector has been performed. The expected number of ionization products at the given ESS condition may be enough if the readout is sufficiently sensitive. The non uniformities of the extraction field can be corrected by hardware corrections, and the distortions of the profile due to space charge effects can be reduced if ions are used and sufficient extraction field is provided to the IPMs. Three readout technologies have been considered and a complete platform has been developed in order to test the different readouts. Two types of IPM have been tested in real beam conditions at IPHI, a $3 \mathrm{MeV}$ proton accelerator. Both strips and MCP were able to measure the beam profile. But approximations to ESS conditions show that the use of MCP is mandatory, therefore the optical IPM is preferred. 
The tests at IPHI were a great opportunity to earn experiences and feedback. From this, an improved IPM design is under development. The IPM is now in two part allowing a quick replacement of the MCP without unmounting the cage. A MCP calibration system is also foreseen to monitor and correct the ageing of MCPs.

The production of the IPMs will be done in an ISO-5 particle free environment to insure the compliance with cryogenic cavities. All IPMs will be qualified at Saclay, according to ESS requirements. First detectors will be delivered at ESS in the beginning of 2020 and will be ready for the first protons on dump.

\section{ACKNOWLEDGMENT}

The authors are grateful to James Storey and his colleagues (CERN, Geneva, Switzerland) for their help with the TimePix detector. We would like to thank Cyril Bachelet and his team (CSNSM, Orsay, France) for their support during the tests at IRMA. We are thankful to our colleagues at IPHI (CEA, Saclay, France) for their assistance and availability during two beam test campaigns. Finally, we thank all people from the ESS collaboration for their fruitful exchanges and support that help us to design the monitors.

\section{REFERENCES}

[1] T. Shea, "Proton beam measurement strategy for the 5 MW European Spallation Source target," in Proc. IBIC2013, 2013, pp. 349-352. [Online]. Available: http://accelconf.web.cern.ch/AccelConf/IBIC2013/ papers/tupc02.pdf

[2] B. Cheymol, A. Jansson, and T. J. Shea, "Wire scanner design for the European Spallation Source," in Proc. IBIC2013, 2013, pp. 830-832. [Online]. Available: http://accelconf.web.cern.ch/AccelConf/IBIC2013/ papers/wepf10.pdf

[3] C. Thomas et al., "Design and implementation of non-invasive profile monitors for the ESS LEBT," in Proc. IBIC'16, 2016, pp. 551-554. [Online]. Available: http://accelconf.web.cern.ch/AccelConf/ ibic2016/papers/tupg80.pdf

[4] W. DeLuca, "Beam Detection using Residual Gas Ionization," in Proc. PAC1969, 1969, pp. 813-822. [Online]. Available: http: //accelconf.web.cern.ch/AccelConf/p69/PDF/PAC1969_0813.PDF

[5] J. Krider, "Residual gas beam profile monitor," Nuclear Instruments and Methods in Physics Research Section A: Accelerators, Spectrometers, Detectors and Associated Equipment, vol. 278, no. 3, pp. $660-$ 663, 1989. [Online]. Available: https://doi.org/10.1016/0168-9002(89) 91188-1

[6] K. Wittenburg, "Experience with the Residual Gas Ionisation Beam Profile Monitors at the DESY Proton Accelerators," in Proc. EPAC1992, 1992, pp. 1133-1135. [Online]. Available: http: //accelconf.web.cern.ch/AccelConf/e92/PDF/EPAC1992_1133.PDF

[7] K. Satou, N. Hayashi, S. Lee, and T. Toyama, "A prototype of Residual Gas Ionization Profile Monitor for J-PARC RCS," in Proc. EPAC2006, 2006, pp. 1163-1165. [Online]. Available: http://accelconf.web.cern.ch/AccelConf/e06/PAPERS/TUPCH065.PDF

[8] T. Giacomini, P. Forck, J. D. Villiers, J. Dietrich, and D. Liakin "Ionization Profile monitors - IPM @ GSI," in Proc. DIPAC2011, 2011, pp. 419-421. [Online]. Available: http://accelconf.web.cern.ch/ AccelConf/DIPAC2011/papers/tupd51.pdf

[9] D. K. Morris et al., "Operational use of ionization profile monitors in the Fermilab Main Injector," in Proc. NA-PAC2011, 2011, pp. 519-521. [Online]. Available: http://accelconf.web.cern.ch/AccelConf/PAC2011/ papers/mop222.pdf

[10] J. Egberts, "IFMIF-LIPAc Beam Diagnostics. Profiling and Loss Monitoring Systems," Theses, Université Paris Sud - Paris XI, Sep. 2012. [Online]. Available: https://tel.archives-ouvertes.fr/tel-00772158

[11] J. Storey et al., "Development of an Ionization Profile Monitor Based on a Pixel Detector for the CERN Proton Synchrotron,' in Proc. IBIC2015, 2015, pp. 470-473. [Online]. Available: http: //accelconf.web.cern.ch/AccelConf/IBIC2015/papers/tupb059.pdf
[12] — "First Results From the Operation of a Rest Gas Ionisation Profile Monitor Based on a Hybrid Pixel Detector," in Proc. IBIC'17, 2017, pp. 318-322. [Online]. Available: http://accelconf.web.cern.ch/ AccelConf/ibic2017/papers/we2ab5.pdf

[13] Tanabashi et al., "Review of particle physics," Phys. Rev. D, vol. 98, p. 030001, Aug 2018. [Online]. Available: https://www.doi.org/10.1103/ PhysRevD.98.030001

[14] S. Kamakura, N. Sakamoto, H. Ogawa, H. Tsuchida, and M. Inokuti, "Mean excitation energies for the stopping power of atoms and molecules evaluated from oscillator-strength spectra," Journal of Applied Physics, vol. 100, no. 6, p. 064905, sep 2006. [Online]. Available: https://www.doi.org/10.1063/1.2345478

[15] J. Weiss and W. Bernstein, "Energy required to produce one ion pair for several gases," Physical Review, vol. 98, no. 6, pp. 1828-1831, jun 1955. [Online]. Available: https://www.doi.org/10.1103/physrev.98.1828

[16] H. Bichsel, D. H. Peirson, J. W. Boring, A. Green, M. Inokuti, and G. Hurst, "Average energy required to produce an ion pair, ICRU Report 31," Journal of the International Commission on Radiation Units and Measurements, vol. 16, no. 2, pp. 18-32, may 1979.

[17] H. Schindler, R. Veenhof et al. (2019) Garfield++ - simulation of tracking detectors. [Online]. Available: http://garfieldpp.web.cern.ch/ garfieldpp/

[18] I. Smirnov, "Modeling of ionization produced by fast charged particles in gases," Nuclear Instruments and Methods in Physics Research Section A: Accelerators, Spectrometers, Detectors and Associated Equipment, vol. 554, no. 1-3, pp. 474-493, dec 2005. [Online]. Available: https://www.doi.org/10.1016/j.nima.2005.08.064

[19] W. W. M. Allison and J. H. Cobb, "Relativistic Charged Particle Identification by Energy Loss," Annual Review of Nuclear and Particle Science, vol. 30, no. 1, pp. 253-298, dec 1980. [Online]. Available: https://www.doi.org/10.1146/annurev.ns.30.120180.001345

[20] R. Wanzenberg. (2010, May) Nonlinear Motion of a Point Charge in the 3D Space Charge Field of a Gaussian Bunch. Hamburg, Germany. [Online]. Available: http://www.desy.de/ mpywar/paper/2010/Internal Report_M_10-01.pdf

[21] C. Thomas, F. Belloni, and J. Marroncle, "Space Charge Studies for the Ionisation Profile Monitors for the ESS Cold Linac,' in Proc. IBIC'16, 2016, pp. 556-559. [Online]. Available: http //accelconf.web.cern.ch/AccelConf/ibic2016/papers/tupg81.pdf

[22] F. Belloni et al., "Space Charge Effects Studies for the ESS Cold Linac Beam Profiler," in Proc. IBIC'18, 2018, pp. 371-374. [Online]. Available: http://accelconf.web.cern.ch/AccelConf/ibic2018/ papers/weoc04.pdf

[23] S. Ramo, "Currents induced by electron motion," Proceedings of the IRE, vol. 27, no. 9, pp. 584-585, sep 1939. [Online]. Available: http://doi.org/10.1109/JRPROC.1939.228757

[24] W. Shockley, "Currents to conductors induced by a moving point charge," J. Appl. Phys., vol. 9, no. 10, p. 635, 1938. [Online]. Available: http://doi.org/10.1063/1.1710367

[25] J. L. Wiza, "Microchannel plate detectors," Nuclear Instruments and Methods, vol. 162, no. 1-3, pp. 587-601, jun 1979.

[26] J. Chaumont, F. Lalu, M. Salome, A.-M. Lamoise, and H. Bernas, "A medium energy facility for variable temperature implantation and analysis," Nuclear Instruments and Methods in Physics Research, vol. 189, no. 1, pp. 193 - 198, 1981. [Online]. Available: https://doi.org/10.1016/0029-554X(81)90145-2

[27] X. Llopart, R. Ballabriga, M. Campbell, L. Tlustos, and W. Wong, "Timepix, a $65 \mathrm{k}$ programmable pixel readout chip for arrival time, energy and/or photon counting measurements," Nuclear Instruments and Methods in Physics Research Section A: Accelerators, Spectrometers, Detectors and Associated Equipment, vol. 581, no. 1, pp. 485 - 494, 2007. [Online]. Available: https://doi.org/10.1016/j.nima.2007.08.079

[28] V. Kraus, M. Holik, J. Jakubek, M. Kroupa, P. Soukup, and Z. Vykydal, "FITPix - fast interface for timepix pixel detectors," Journal of Instrumentation, vol. 6, no. 01, pp. C01079-C01079, jan 2011. [Online]. Available: https://doi.org/10.1088/1748-0221/6/01/c01079

[29] P.-Y. Beauvais et al., "Status report on the saclay high-intensity proton injector project (IPHI)." in Proc. EPAC2000, 2000, pp. 283285. [Online]. Available: http://accelconf.web.cern.ch/AccelConf/e00/ PAPERS/THOAF202.pdf

[30] R. Gobin et al., "High intensity beam production at CEA/Saclay for the IPHI project," in Proc. ECRIS2016, 2016, pp. 83-85. [Online]. Available: http://accelconf.web.cern.ch/AccelConf/ecris2016/ doi/JACoW-ECRIS2016-WEPP01.html

[31] F. Sene et al., "Increase of IPHI Beam Power at CEA Saclay," in Proc. IPAC'18, 2018, pp. 694-696. [Online]. Available: http://accelconf.web. cern.ch/AccelConf/ipac2018/doi/JACoW-IPAC2018-TUPAF016.html 